\title{
Der Chirurg
}

Erratum

Chirurg 2021 · 92:70

https://doi.org/10.1007/s00104-020-01318-0

Online publiziert: 18 . November 2020

(c) Springer Medizin Verlag $\mathrm{GmbH}$, ein Teil von Springer Nature 2020

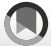

Andreas Proske' $\cdot$ Björn-Christian Link ${ }^{2} \cdot$ Frank J. P. Beeres $^{2} \cdot$ Sven Nebelung ${ }^{3} \cdot$ Bernd Füchtmeier ${ }^{1} \cdot$ Matthias Knobe $^{2}$

${ }^{1}$ Klinik für Orthopädie, Unfallchirurgie und Sportmedizin, Krankenhaus Barmherzige Brüder Regensburg, Regensburg, Deutschland

${ }^{2}$ Klinik für Orthopädie und Unfallchirurgie, Luzerner Kantonsspital, Luzern, Schweiz

${ }^{3}$ Institut für diagnostische und interventionelle Radiologie, UniversitätsklinikumDüsseldorf, Düsseldorf Deutschland

\section{Erratum zu: Weiterbildung unter der Lupe (Teil 2) - Wie bereiten sich Weiterbildungsassistenten auf Notfalloperationen vor?}

\section{Erratum zu:}

\section{Chirurg 2020}

https://doi.org/10.1007/s00104-020-

01286-5

Der Koautor, Herr PD Dr. Beeres, hat die Vornamensinitialen F.J.P. In der ursprünglichen Publikation wurden die Vornamensinitialen J.P. vergessen. Die Autoren bitten den Fehler zu entschuldigen.

\section{Korrespondenzadresse}

\section{Dr. med. Andreas Proske, MME}

Klinik für Orthopädie, Unfallchirurgie und Sportmedizin, Krankenhaus Barmherzige Brüder Regensburg

Prüfeninger Str. 86, 93049 Regensburg,

Deutschland

andreas.proske@barmherzige-regensburg.de 